\title{
X-ray Fourier Ptychography for Out-Of-Focus Measurements
}

\author{
Klaus Wakonig ${ }^{a, b}$, Ana Diaz ${ }^{a}$, Sabina Chiriotti ${ }^{a}$, Anna Bergamaschi ${ }^{a}$, Anne Bonnin $^{a}$, Marco \\ Stampanoni ${ }^{a, b}$, and Andreas Menzel $^{a}$ \\ ${ }^{a}$ Paul Scherrer Institute, Forschungsstrasse 111, 5232 Villigen PSI, Switzerland \\ ${ }^{b} \mathrm{ETH}$ and University of Zürich, Institute for Biomedical Engineering, 8093 Zürich, \\ Switzerland
}

\begin{abstract}
Transmission microscopes have become a valuable tool for hard X-ray imaging. They allow even complex in situ and operando setups to be realized. However, the objective lens, typically a Fresnel zone plate with a high numerical aperture, is commonly a limiting factor. The small working distance as well as the low efficiency of Fresnel zone plates with high numerical apertures restrict setups either to accommodate specific sample environments or to provide high resolution. Lensless imaging techniques, e.g. ptychography, do not suffer from such adverse effects of Fresnel zone plates. Consequently, they are frequently used for high-resolution X-ray imaging. A recently developed method, X-ray Fourier ptychography aims to combine the benefits of both techniques. It has been shown to provide quantitative high-resolution imaging whilst keeping large working distances for in situ and operando setups. This is achieved by acquiring multiple images with a full-field transmission microscope, each at a different lateral position of the Fresnel zone plate. Moving the objective off the optical axis varies the frequency content for the acquisitions. The resulting dataset is numerically combined using well-established phase retrieval algorithms to recover a complex-valued representation of the sample. Here, we demonstrate how Fourier ptychographic phase retrieval can further be used to mitigate artifacts caused by samples that were placed out of focus, as well as misaligned optical elements. Employing a similar approach to increase the contrast in case of weakly absorbing specimens is also envisioned.
\end{abstract}

Keywords: X-ray imaging, Fourier ptychography, MÖNCH detector, alignment, phase constrast

\section{INTRODUCTION}

With the increase in flux of modern X-ray sources and the improvements of large and fast 2D detectors, X-ray imaging has evolved to a widely used technique in various research fields. X-ray microscopy methods using higher energies could potentially benefit from the smaller wavelength and the increased penetration depth. However, in particular for imaging modalities using hard X-rays, the achievable resolution remains limited by the objective lens's capability to capture large diffraction angles. This is commonly quantified by the numerical aperture $N A$, which is related to the refractive index $n$ and diffraction angle $\theta$ via,

$$
N A=n \cdot \sin (\theta) .
$$

In visible light, where microscopes can rely on almost perfect optics, the $N A$ can reach values even larger than 1. Yet, diffractive optics for hard X-rays like Fresnel zone plates commonly reach a numerical aperture of only 0.001. It is for this reason that in this energy regime, lens-less imaging methods such as ptychography have been of particular interest. ${ }^{1}$ However, it has been shown that ptychographic principles can be translated from lens-less imaging to lens-based techniques, alleviating limitations imposed by the objective. ${ }^{2-4}$

In conventional ptychography, the sample is scanned along a predefined 2D pattern. The step size of the movement is chosen such that the illuminating cones of adjacent scan positions are overlapping. At each position, a diffraction pattern is recorded, typically in the far-field regime. The measured intensity $I_{j}(\mathbf{q})$ of the

All correspondence should be addressed to:

K. Wakonig: klaus.wakonig@psi.ch

A. Menzel: andreas.menzel@psi.ch

\footnotetext{
X-Ray Nanoimaging: Instruments and Methods IV, edited by Barry Lai,

Andrea Somogyi, Proc. of SPIE Vol. 11112, 111120I - (c) 2019 SPIE

CCC code: $0277-786 X / 19 / \$ 21 \cdot$ doi: $10.1117 / 12.2528905$
}

Proc. of SPIE Vol. 11112 111120I-1 
$j^{\text {th }}$ scan position can then be written as the Fourier transform of the wave past the specimen, also known as the exit wave $\Psi_{j}(\mathbf{r}),{ }^{5,6}$

$$
I_{j}(\mathbf{q})=\left|\mathcal{F}\left(\Psi_{j}(\mathbf{r})\right)\right|^{2},
$$

with $\mathbf{r}$ and $\mathbf{q}$ being the transverse real-space and reciprocal-space coordinate vectors, respectively.

Iterative reconstruction algorithms are used to recover the exit wave from the acquired instensity patterns. ${ }^{6-9}$ Further, the complex illumination wave front $P\left(\mathbf{r}-\mathbf{r}_{j}\right)$ and an object transmissivity $O(\mathbf{r})$, can be reconstructed simultaneously by decomposing the exit wave into, $, 6,10,11$

$$
\Psi_{\mathrm{j}}(\mathbf{r})=P\left(\mathbf{r}-\mathbf{r}_{\mathrm{j}}\right) O(\mathbf{r}) .
$$

In case of lens-based imaging modalities, an objective lens is placed between sample and detector, resulting in a magnified image of specimen at the detector plane. Assuming a flat illumination of the sample, the propagated wave directly after the lens plane can be written by means of the complex-valued spectrum $S\left(\mathbf{q}^{\prime}\right)$ and the pupil function $\hat{P}\left(\mathbf{q}^{\prime}\right)$,

$$
\hat{\Psi}\left(\mathbf{q}^{\prime}\right)=\mathcal{F}(\Psi(\mathbf{r})) \hat{P}\left(\mathbf{q}^{\prime}\right)=S\left(\mathbf{q}^{\prime}\right) \hat{P}\left(\mathbf{q}^{\prime}\right) .
$$

By varying the angle of the sample's illumination, the spectrum can be scanned across a fixed pupil function. This resembles an analog form of Eq. 3 in Fourier space,

$$
\hat{\Psi}_{j}\left(\mathbf{q}^{\prime}\right)=S\left(\mathbf{q}^{\prime}-\mathbf{q}_{\mathbf{j}}^{\prime}\right) \hat{P}\left(\mathbf{q}^{\prime}\right) .
$$

Similar to Eq. 2, the measured intensity can be written as the propagated exit wave,

$$
\hat{I}_{j}\left(\mathbf{r}^{\prime}\right)=\left|\mathcal{F}\left(\hat{\Psi}_{j}\left(\mathbf{q}^{\prime}\right)\right)\right|^{2} .
$$

As the scanning range of the spectrum can be significantly larger than the finite length of the pupil function, limitations imposed by the objective lens can be lessened. Moreover, the same reconstruction algorithms as for conventional ptychography can be applied to reconstruct the sample's spectrum and the pupil function simultaneously.

Due to its similarities with conventional ptychography, such scanning techniques are labeled Fourier ptychography and have been demonstrated in visible light. ${ }^{2,3}$ Recently, we have shown that such concepts can be translated from visible light to the X-ray regime to provide a quantitative high-resolution imaging modality, with superior phase and absorption contrast, than otherwise gained with a transmission X-ray microscope (TXM). ${ }^{4}$

In general, a transmission X-ray microscope relies on a perfectly aligned objective lens with a well-defined distance between sample, objective lens and detector. Any discrepancy will lead to an additional phase factor in Eq. 4 and thus directly affect the acquired data, Eq. 6, resulting in a reduced image quality and resolution. However, in addition to the aforementioned ptychographic benefits compared to a standard transmission X-ray microscope, reconstructing the complex-valued wavefront allows for further image processing. In this work, we show that X-ray Fourier ptychography is able to recover a focused image, even though the sample was placed out of focus.

Such abilities may not only improve the image quality, but also reduce the requirements of a meticulous alignment. Moreover, as the phase contrast in hard X-ray imaging can be orders of magnitude larger than the absorption contrast, weakly absorbing samples may further benefit from a propagation-based contrast enhancement.

\section{RESULTS}

To demonstrate these abilities, we set up a transmission X-ray microscope at the cSAXS beamline, Swiss Light Source, Villigen, Switzerland. In contrast to Fourier ptychography in visible light, where the spectrum can conveniently be scanned by means of changing the illumination direction, we performed X-ray Fourier ptychography by scanning the objective lens transversal to the beam direction. ${ }^{4}$ 


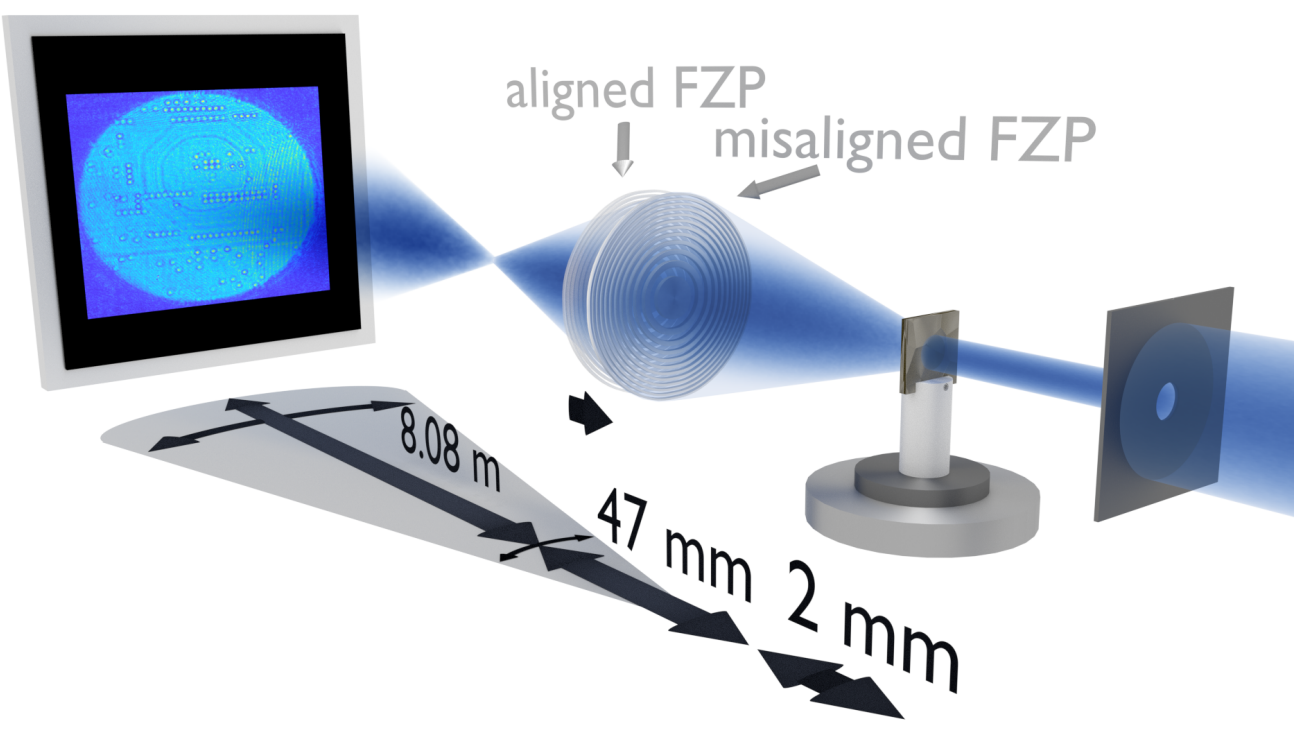

Figure 1. Setup of X-ray Fourier ptychography at the cSAXS beamline. Instead of changing the illumination direction, X-ray Fourier ptychography can be performed by scanning the objective lens, in this case a Fresnel zone plate. The objective was placed at a distance of $47 \mathrm{~mm}$ from the sample, approximately $2 \mathrm{~mm}$ out of focus. As a result, propagationbased phase contrast increases the visibility of features.

Similarly to previous measurements, ${ }^{4}$ we used a $30 \mu \mathrm{m}$ pinhole to define a coherent illumination of the sample, an application-specific integrated circuit (ASIC). ${ }^{4,12}$ Acquisitions were performed with a photon energy of $8.7 \mathrm{keV}$, corresponding to a wavelength of $\lambda=1.4 \AA$. A Fresnel zone plate with an outermost zone width of $70 \mathrm{~nm}$ and a diameter of $100 \mu \mathrm{m}$ was used as objective lens. It was mounted on a piezo stage for high position accuracy. The depth-of-field was calculated to $68 \mu \mathrm{m}$. Images were collected using a MÖNCH detector ${ }^{13,14}$ with a physical pixel size of $25 \mu \mathrm{m}$. The detector was placed at a distance of $8.076 \mathrm{~m}$ downstream of the sample. After performing a reference scan with an aligned zone plate, we moved the objective lens by approximately $2 \mathrm{~mm}$, i.e. 30 times the depth-of-field, towards the sample and repeated the scan (Fig. 1).

For each dataset, 362 images were taken by scanning the FZP along a Fermats spiral ${ }^{15}$ with overall range of $80 \mu \mathrm{m}$ in both directions. Due to the limited size of the detector, the movements of the objective had to be followed by movements of the detector. Raw data frames, Fig. 2, were interpolated from a physical $25 \mu \mathrm{m}$ detector pixel pitch to a sub-pixel resolution of $6.25 \mu \mathrm{m} .{ }^{4,13}$ Images in Fig. 2a-b were taken close to the optical axis. The data taken with an aligned objective lens (Fig. 2a) has a significantly lower contrast due to the object's poor absorption contrast. By moving the objective out of focus, propagation-based phase contrast increases the visibility of features that were barely visible otherwise.

Such effects are reduced when working far away from the optical axis, shown in Fig. 2c-d, as the visibility of features in the aligned setup is already improved by edge enhancement, due to the high frequency part of the spectrum probed at these positions. Before reconstructing the spectrum, raw-data frames were aligned using a routine described in Wakonig et al., 2019. ${ }^{4,16}$

Two independent measurements taken with the misaligned setup were reconstructed using 1000 iterations of least-squares maximum-likelihood (LSQ-ML) ${ }^{9}$ Fig. 3. The obtained reconstruction of the sample's spectrum was backpropagated to the expected sample plane of an in-focus setup. The misalignment of the FZP was corrected by propagating the object's transmissivity by $2 \mathrm{~mm}$. To eliminate small displacements between the reconstructions, the results were sub-pixel aligned ${ }^{16}$ before calculating a Fourier ring correlation (FRC). ${ }^{17}$ The crossing of a 1-bit threshold, shown in Fig. 4, indicates a resolution of $47 \mathrm{~nm}$, and thus significantly below the Rayleigh resolution limit of $85 \mathrm{~nm}$ for a perfectly aligned setup. Moreover, a comparison with previous measurements of the same sample show that there is no indication of any loss in image quality or resolution. ${ }^{4}$ 

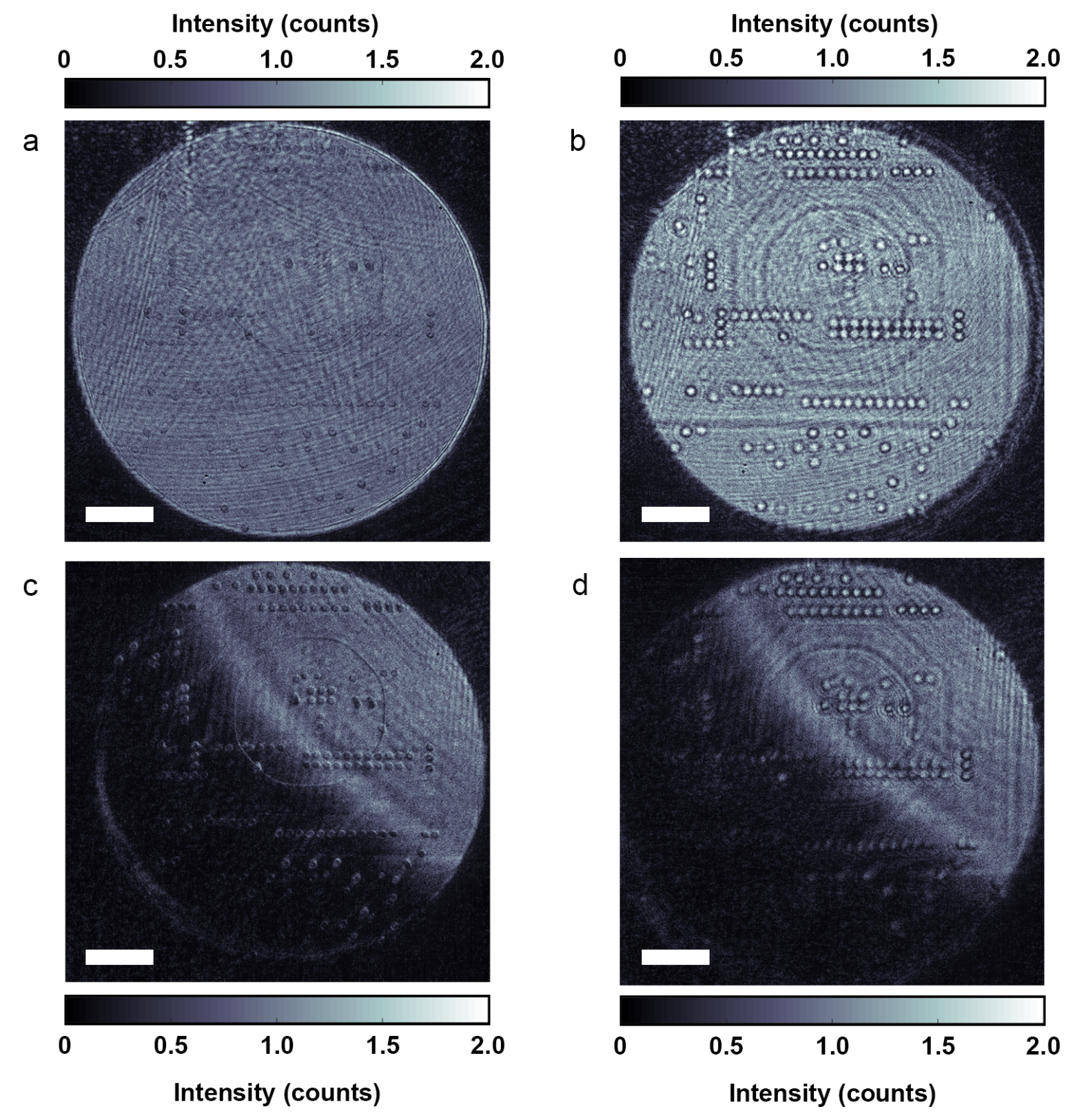

Figure 2. Raw data frames after interpolating to a pixel size of $6.25 \mu \mathrm{m}$. (a-b) Raw data frames after moving the FZP vertically by approximately $25 \mu \mathrm{m}$ away from the optical axis, with an aligned FZP (a) and a misaligned FZP (b). (c-d) Raw data frames after moving the FZP horizontally and vertically by approximately $40 \mu \mathrm{m}$ away from the optical axis, with an aligned FZP (c) and a misaligned FZP (d). Due to edge enhancement, differences in contrast that were prominent close to the optical axis are significantly reduced. However, propagation-based artifacts are still clearly visible. Scale bars: $5 \mu \mathrm{m}$. 

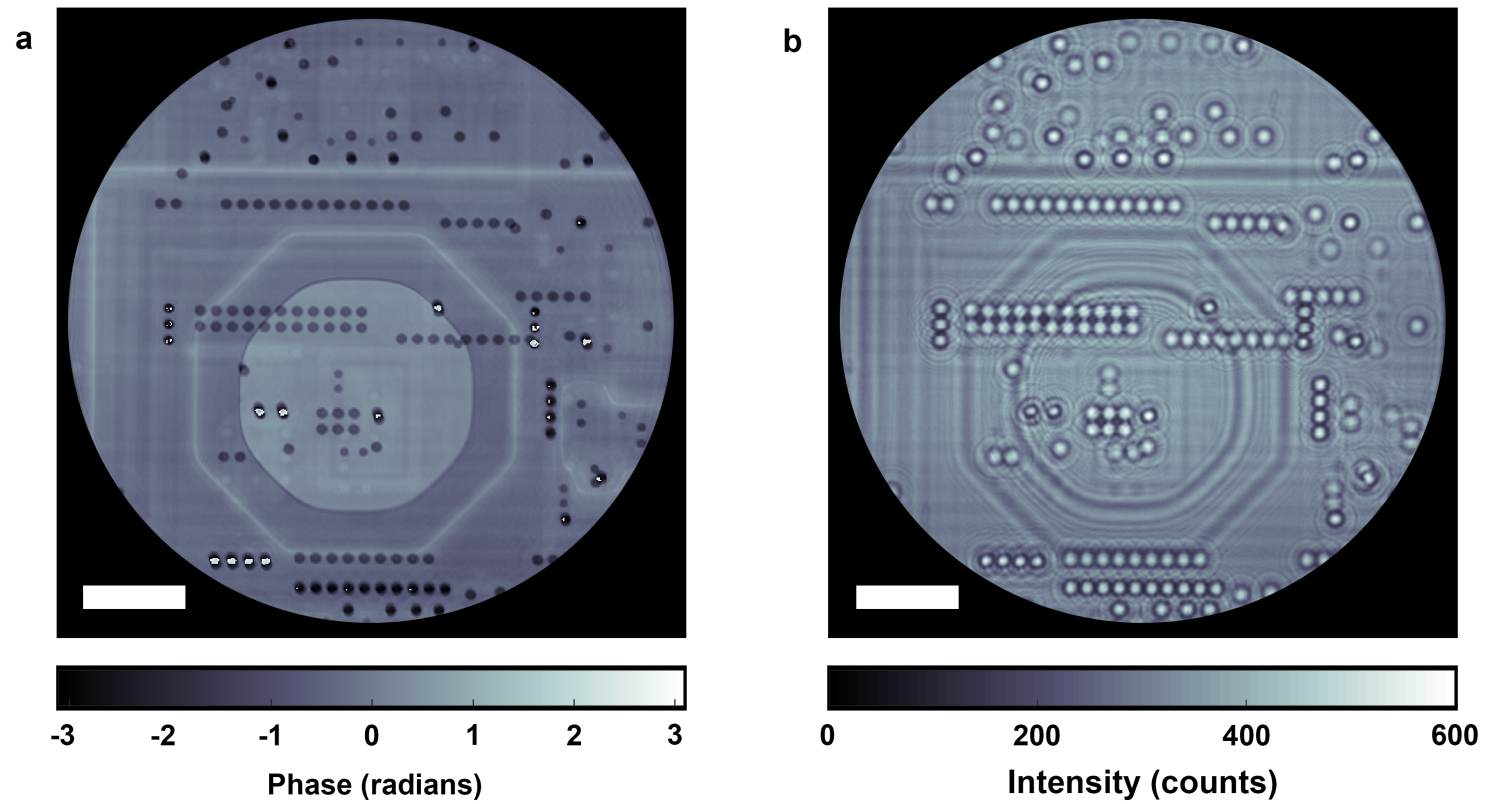

Figure 3. (a) Phase of the backpropagated spectrum. (b) Aligned and summed-up raw data. By summing-up the raw data intensities, a measurement with a standard transmission X-ray microscope can be simulated. The result highlights how a misplacement by 30 times the depth-of-focus would affect measurements with a TXM. Scale bars: $5 \mu \mathrm{m}$.

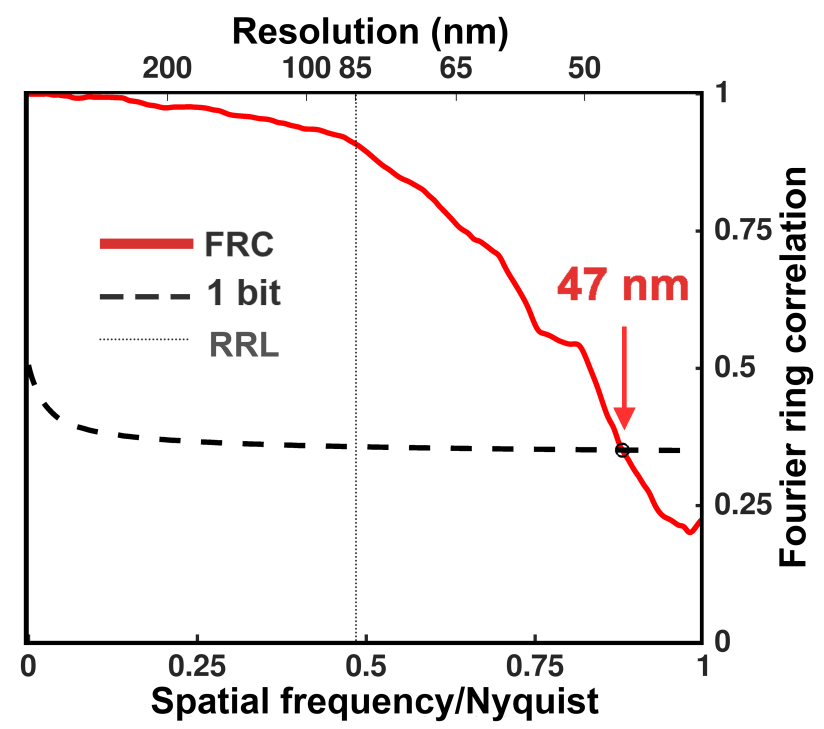

Figure 4. Resolution estimation by means of a Fourier ring correlation. Comparing the Fourier ring correlation to the 1-bit criterion results in a resolution of $47 \mathrm{~nm}$ and thus significantly below the Rayleight resolution limit (RRL) of 85 $\mathrm{nm}$ for a Fresnel zone plate with an outermost zone width of $70 \mathrm{~nm}$ and a diameter of $100 \mu \mathrm{m}$, indicated by a dotted vertical line. 


\section{CONCLUSION}

Beyond the improvements in image quality, resolution and a quantitative reconstruction, X-ray Fourier ptychography can be more robust against misalignment artifacts compared to a standard transmission X-ray microscope. Here, we have shown how even a propagation of 30 times the depth-of-focus can be reliably corrected for.

Moreover, the improved contrast may prove beneficial for weakly absorbing specimens for which the low visibility of features would otherwise hinder a meticulous alignment of sample and objective lens.

In the prospect of the increased coherent flux at $4^{\text {th }}$ generation synchrotrons, these results highlight that coherent methods like X-ray Fourier ptychography may prove to contribute a valuable complement to their incoherent counterparts, which they outperform in terms of image quality, resolution and a quantitative phase contrast.

\section{ACKNOWLEDGMENTS}

We would like to thank Manuel Guizar-Sicairos, Mariana Verezhak and Xavier Donath for their support at the cSAXS beamline. Moreover, we acknowledge financial support from the Swiss National Science Foundation (SNF), grant number 166304 (K.W.).

\section{REFERENCES}

[1] Pfeiffer, F., "X-ray ptychography," Nature Photonics 12, 9-17 (jan 2018).

[2] Zheng, G., Horstmeyer, R., and Yang, C., "Wide-field, high-resolution Fourier ptychographic microscopy," Nature Photonics 7(9), 739-745 (2013).

[3] Horstmeyer, R. and Yang, C., "A phase space model of Fourier ptychographic microscopy," Optics Express 22(1), 338 (2014).

[4] Wakonig, K., Diaz, A., Bonnin, A., Stampanoni, M., Bergamaschi, A., Ihli, J., Guizar-Sicairos, M., and Menzel, A., "X-ray Fourier ptychography," Science Advances 5, eaav0282 (feb 2019).

[5] Rodenburg, J. M. and Faulkner, H. M. L., "A phase retrieval algorithm for shifting illumination," Applied Physics Letters 85(20), 4795-4797 (2004).

[6] Thibault, P., Dierolf, M., Menzel, A., Bunk, O., David, C., and Pfeiffer, F., "High-resolution scanning x-ray diffraction microscopy.," Science (New York, N.Y.) 321, 379-382 (2008).

[7] Maiden, A. M. and Rodenburg, J. M., "An improved ptychographical phase retrieval algorithm for diffractive imaging," Ultramicroscopy 109(10), 1256-1262 (2009).

[8] Thibault, P. and Guizar-Sicairos, M., "Maximum-likelihood refinement for coherent diffractive imaging," New Journal of Physics 14, 063004 (2012).

[9] Odstrčil, M., Menzel, A., and Guizar-Sicairos, M., "Iterative least-squares solver for generalized maximumlikelihood ptychography," Optics Express 26, 3108 (feb 2018).

[10] Rodenburg, J. M. and Bates, R. H. T., "The theory of super-resolution electron microscopy via Wignerdistribution deconvolution," Philosophical Transactions of the Royal Society of London. Series A: Physical and Engineering Sciences 339, 521-553 (jun 1992).

[11] Thibault, P., Dierolf, M., Bunk, O., Menzel, A., and Pfeiffer, F., "Probe retrieval in ptychographic coherent diffractive imaging," Ultramicroscopy 109, 338-343 (2009).

[12] Guizar-Sicairos, M., Johnson, I., Diaz, A., Holler, M., Karvinen, P., Stadler, H.-C., Dinapoli, R., Bunk, O., and Menzel, A., "High-throughput ptychography using Eiger-scanning X-ray nano-imaging of extended regions," Optics Express 22(12), 14859 (2014).

[13] Cartier, S., Kagias, M., Bergamaschi, A., Wang, Z., Dinapoli, R., Mozzanica, A., Ramilli, M., Schmitt, B., Brückner, M., Fröjdh, E., Greiffenberg, D., Mayilyan, D., Mezza, D., Redford, S., Ruder, C., Schädler, L., Shi, X., Thattil, D., Tinti, G., Zhang, J., and Stampanoni, M., "Micrometer-resolution imaging using MÖNCH: Towards G2-less grating interferometry," Journal of Synchrotron Radiation 23, 1462-1473 (nov 2016). 
[14] Ramilli, M., Bergamaschi, A., Andrae, M., Brückner, M., Cartier, S., Dinapoli, R., Fröjdh, E., Greiffenberg, D., Hutwelker, T., Lopez-Cuenca, C., Mezza, D., Mozzanica, A., Ruat, M., Redford, S., Schmitt, B., Shi, X., Tinti, G., and Zhang, J., "Measurements with MÖNCH, a $25 \mu \mathrm{m}$ pixel pitch hybrid pixel detector," Journal of Instrumentation 12, C01071-C01071 (jan 2017).

[15] Huang, X., Yan, H., Harder, R., Hwu, Y., Robinson, I. K., and Chu, Y. S., "Optimization of overlap uniformness for ptychography," Optics Express 22(10), 12634-12644 (2014).

[16] Guizar-Sicairos, M., Thurman, S. T., and Fienup, J. R., "Efficient subpixel image registration algorithms.," Optics Letters 33(2), 156-158 (2008).

[17] van Heel, M. and Schatz, M., "Fourier shell correlation threshold criteria," Journal of Structural Biology 151, 250-262 (sep 2005). 